\title{
The total number and mass of SARS-CoV-2 virions
}

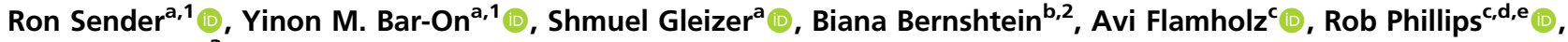 \\ and Ron Miloa,
}

\begin{abstract}
${ }^{a}$ Department of Plant and Environmental Sciences, Weizmann Institute of Science, Rehovot 7610001, Israel; ${ }^{b}$ Department of Molecular Genetics, Weizmann Institute of Science, Rehovot 7610001, Israel; 'Division of Biology and Biological Engineering, California Institute of Technology, Pasadena, CA 91125; ${ }^{\mathrm{d} D e p a r t m e n t}$ of Physics, California Institute of Technology, Pasadena, CA 91125; and ${ }^{\mathrm{e} C h a n}$ Zuckerberg Biohub, San Francisco, CA 94158
\end{abstract}

Edited by Ken A. Dill, Stony Brook University, Stony Brook, NY, and approved May 10, 2021 (received for review December 13, 2020)

Quantitatively describing the time course of the severe acute respiratory syndrome coronavirus 2 (SARS-CoV-2) infection within an infected individual is important for understanding the current global pandemic and possible ways to combat it. Here we integrate the best current knowledge about the typical viral load of SARS-CoV-2 in bodily fluids and host tissues to estimate the total number and mass of SARS-CoV-2 virions in an infected person. We estimate that each infected person carries $10^{9}$ to $10^{11}$ virions during peak infection, with a total mass in the range of $1 \mu \mathrm{g}$ to $100 \mu \mathrm{g}$, which curiously implies that all SARS-CoV-2 virions currently circulating within human hosts have a collective mass of only $0.1 \mathrm{~kg}$ to $10 \mathrm{~kg}$. We combine our estimates with the available literature on host immune response and viral mutation rates to demonstrate how antibodies markedly outnumber the spike proteins, and the genetic diversity of virions in an infected host covers all possible single nucleotide substitutions.

COVID-19 | variants of concern | viral biomass | viral load | genetic diversity

$\mathbf{E}_{\mathrm{a}}^{\mathrm{s}}$ stimating key biological quantities such as the total number and mass of cells in our body or the biomass of organisms in the biosphere in absolute units improves our intuition and understanding of the living world (1-4). Such a quantitative perspective could help the current intensive effort to study and model the spread of the COVID-19 pandemic. We have recently compiled quantitative data at the virus level as well as at the community level to help communicate state-of-the-art knowledge about the severe acute respiratory syndrome coronavirus 2 (SARS-CoV-2) to the public and researchers alike and provide them with a quantitative toolkit to think about the pandemic (5). Here we leverage such quantitative information to estimate the total number and mass of SARS-CoV-2 virions present in an infected individual during the peak of the infection.

Viral loads are commonly measured in two distinct ways: counting viral RNA genomes by qRT-PCR and measuring the number of infectious units in tissue culture (6). The second approach incubates susceptible mammalian cells with dilutions of a patient sample to determine the amount of sample required to kill $50 \%$ of the cells. This value is used to back-calculate the infectious titer in the sample in units of " $50 \%$ tissue culture infective dose" or TCID 50 [for example, by the Reed and Muench method (7)]. The TCID 50 is analogous (and often quantitatively similar) to the plaque-forming units (PFU) assay. Here, we refer to TCID $_{50}$ and PFU more generally as "infectious units." As these two measurement modalities (RNA genome copies and infectious units) differ in reported values and interpretation - one method measuring the number of RNAs, the other measuring the number of infectious units-we report and compare estimates stemming from both approaches.

Estimate of the Number of Virions in an Infected Individual To estimate the total number of virions present in an infected individual at the peak of infection, we rely on three studies which measured the concentration of SARS-CoV-2 genomic RNA in the tissues of infected rhesus macaques $2 \mathrm{~d}$ to $4 \mathrm{~d}$ after inoculation with the virus (8-10). Viral concentrations were measured in samples of all the relevant tissues of the respiratory, digestive, and immune systems, and values are given in units of genome copies per gram tissue. We use values measured in rhesus macaques, as they are the closest organism to humans where such comprehensive data are available. Using these measurements, we estimate the total number of virions by multiplying the concentration of viral genomes in each tissue by the total tissue mass $(11,12)$. We assume that each genome is associated with a virion (i.e., the ratio of virions to genome copies $\left.F_{\text {virions to } R N A \text { copies }} \approx 1\right)$. In case where a large fraction of the viral RNA copies are present as "naked" RNA (not encapsulated inside viral particles), using viral RNA copies as a proxy for the number of viral particles could lead to an overestimate. We expand on this source of uncertainty in the discussion. As seen in Fig. 1, the lungs are the largest of these tissues on a mass basis ( $M_{\text {lungs }} \approx 1 \mathrm{~kg}$ ). Lungs were also found to harbor the highest concentration of viral RNA $\left(C_{\text {genome copies }}^{\text {lung }} \approx 10^{6}\right.$ to $10^{8}$ [RNA copies per g]; see SI Appendix for full details and comparison with additional sources), and we therefore estimate that virions in the lungs are the dominant contributor to the total number of virions in the body during peak infection, with

$$
\begin{aligned}
& C_{\text {genome copies }}^{\text {lungs }} \times M_{\text {lungs }} \times F_{\text {virions to } R N A \text { copies }}=N_{\text {virions }} \\
& \begin{aligned}
10^{6} \text { to } 10^{8}[\mathrm{RNA} \text { copies per } \mathrm{g}] \times 1,000[\mathrm{~g}] \\
\times 1[\text { virions to RNA copies] } \\
=10^{9} \text { to } 10^{11} \text { [virions]. }
\end{aligned}
\end{aligned}
$$

\section{Significance}

Knowing the absolute numbers of virions in an infection promotes better understanding of disease dynamics and response of the immune system. Here we use current knowledge on the concentrations of virions in infected individuals to estimate the total number and mass of SARS-CoV-2 virions in an infected person. Although each infected person carries an estimated 1 billion to 100 billion virions during peak infection, their total mass is no more than $0.1 \mathrm{mg}$. This curiously implies that all SARS-CoV-2 virions currently in all human hosts have a mass of between $100 \mathrm{~g}$ and $10 \mathrm{~kg}$. Combining the known mutation rate and our estimate of the number of infectious virions, we quantify the formation rate of genetic variants.

Author contributions: R.S., Y.M.B.-O., R.P., and R.M. designed research; R.S., Y.M.B.-O., S.G., B.B., A.F., R.P., and R.M. performed research; R.S., Y.M.B.-O., S.G., B.B., A.F., R.P., and R.M. analyzed data; and R.S., Y.M.B.-O., S.G., B.B., A.F., R.P., and R.M. wrote the paper.

The authors declare no competing interest.

This article is a PNAS Direct Submission.

This open access article is distributed under Creative Commons Attribution License 4.0 (CC BY).

${ }^{1}$ R.S. and Y.M.B.-O. contributed equally to this work.

${ }^{2}$ Present address: Ragon Institute of MGH, MIT and Harvard, Cambridge, MA 02138.

${ }^{3}$ To whom correspondence may be addressed. Email: ron.milo@weizmann.ac.il.

This article contains supporting information online at https://www.pnas.org/lookup/suppl/ doi:10.1073/pnas.2024815118/-/DCSupplemental.

Published June 3, 2021. 


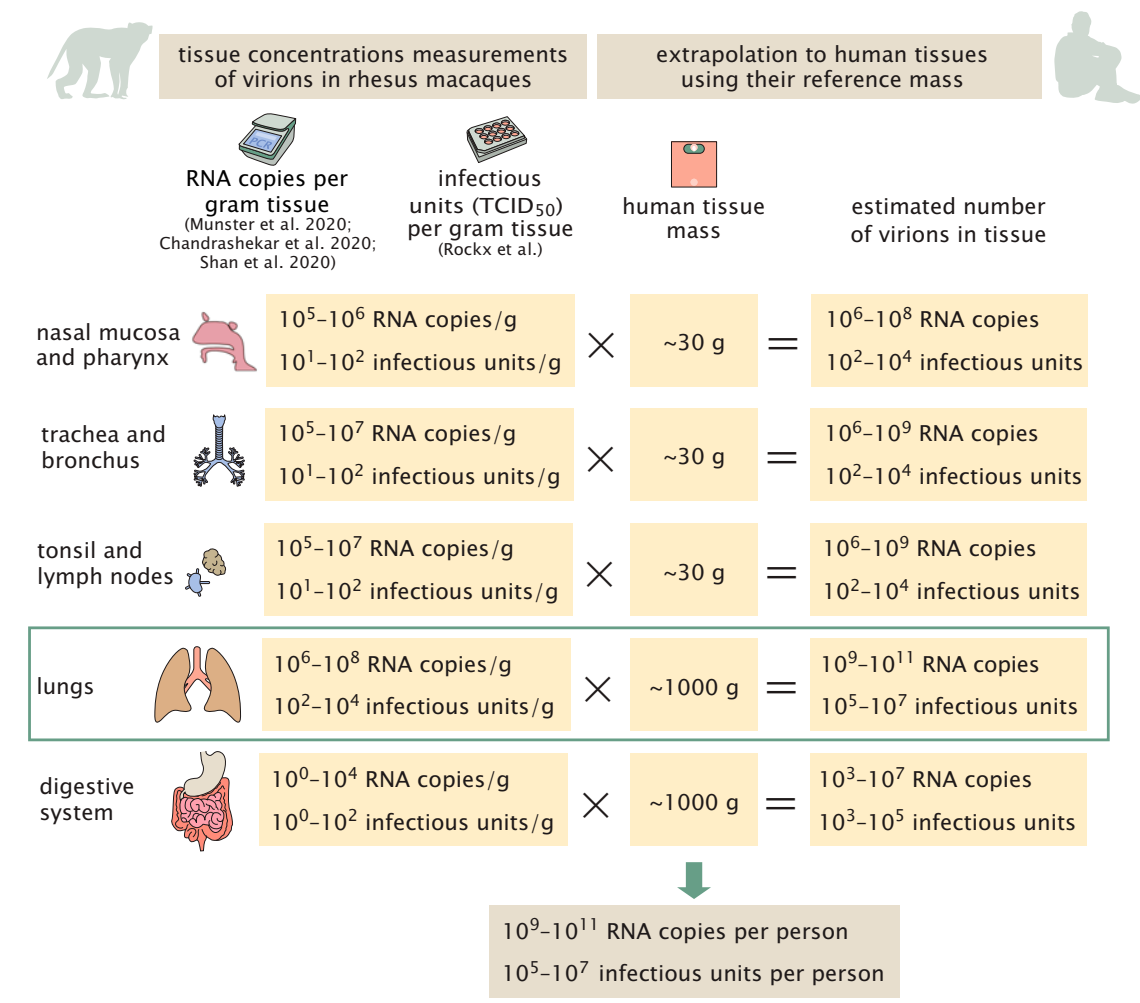

Fig. 1. A schematic representation of the estimate of the number of virions in an infected individual. The estimate is made using the viral load measured in a gram of rhesus macaque tissue $(8-10,13)$ multiplied by the mass of human tissues in a reference adult person with a total body weight of 70 kg $(11)$. In the digestive tract, the concentrations are close to the detection limit. We assume the number of virions is similar to the number of RNA copies.

Other tissues, like the nasal mucosa, larynx, bronchial tree, and adjacent lymph nodes, all have a combined mass of $100 \mathrm{~g}(12)$ and maximal concentrations of $10^{6}$ to $10^{7}$ RNA copies per $\mathrm{mL}$ and hence contribute, at most, an additional $10 \%$ to an estimate based solely on the lungs (Fig. 1).

Another study (13) measured concentrations of infectious virus in tissues of infected rhesus macaques $4 \mathrm{~d}$ after inoculation, using cell culture methods. This study reports measurements in units of $\mathrm{TCID}_{50}$. The maximal values in these units are much smaller, on the order of $10^{3}$ TCID $_{50}$ per mL to $10^{4}$ TCID $_{50}$ per $\mathrm{mL}$ for lung tissue. Combining these measurements with the volume of adult human lung tissue $(\approx 1 \mathrm{~L})$, we get an estimate of $10^{5}$ to $10^{7}$ infectious units in an adult, compared with $10^{9}$ to $10^{11}$ RNA copies, estimated from the other studies (Fig. 1). These data suggest a difference of roughly four orders of magnitude between RT-PCR measurements of viral RNA and tissue culture measurements of viral titers in TCID $_{50}$ units. To check the consistency of this result with the published literature, we collected 13 studies that measured SARS-CoV-2 viral RNA copies as well as TCID $_{50}$ or PFU in monkeys and human samples (SI Appendix). The characteristic ratio between RNA copy measurements and $\mathrm{TCID}_{50}$ measurements is about four orders of magnitude but can vary between three and five orders of magnitude. We attend to this seeming discrepancy between viral genomic copies and infectious units in Discussion. We continue to analyze what can be inferred from the evidence that the total number of virions in an infected individual during peak infection is $10^{9}$ to $10^{11}$, and the number of infectious units is $10^{5}$ to $10^{7}$.

While the estimates were performed using a reference value for the lung mass taken from adult men, they can be generalized to the case of women and children. We rely on the multiplication of the viral concentration in the lungs and the total mass of the lungs. Reference values for the lung mass show a value smaller by $20 \%$ for women, and 25 to $75 \%$ smaller for children aged 5 y to $15 \mathrm{y}(12)$. Although COVID-19 is known to affect adult men more than women and children $(14,15)$, there is scarce information regarding difference in viral concentrations across gender and age. One preprint (16) suggests that viral concentration in children is lower by up to an order of magnitude, but the change they measured is not consistent across the entire age range. Assuming the change in measured viral load represents a similar change in viral concentration in the lung tissue, and combining the concentrations with the reduced lung mass, we get that the number of virions in an infected woman is similar to that estimated for men (i.e., of the same order), and that an infected child is probably carrying an order of magnitude fewer virions.

In addition to analyzing the state of an infected individual during peak infection, we can also estimate the total number of virions and infectious units produced over the course of an infection, as well as the rate of virion production inside a human host. To estimate the total number of virions produced during an infection, we consider the viral load curve as a function of the time since infection. The total production of virions can be estimated by the area under the viral load curve divided by the reciprocal of the viral clearance rate (equivalent to the viral residence time; the integral has units of [virions $\times$ time], and so it needs to be divided by a residence time to get units of [virions]). Using a previously published model of exponential growth and decay (17), we analytically calculated the area under the curve. Dividing by estimates for the inverse of the viral clearance rate (equivalent to the residence time) (18-20) gives an estimated total production of $3 \times 10^{9}$ to $3 \times 10^{12}$ virions, or $3 \times 10^{5}$ to $3 \times$ $10^{8}$ infectious units over the complete course of a characteristic infection (see SI Appendix for details). Thus, the ratio between the total production of virions to their peak number is in the range of 3 to 30 . 
To understand the meaning of the above ratio, it is helpful to consider the shape of the viral load curve. Typical patients' viral loads increase sharply until reaching a peak, after which they decrease rapidly. As the load curve is steep and the extracellular resident time of virions is not very long [estimated to be $1 \mathrm{~h}$ to $10 \mathrm{~h}(18-20)]$, a large fraction of all virions produced must be produced near the peak of infection. Therefore, the cumulative production of virions in the $1 \mathrm{~d}$ to $3 \mathrm{~d}$ near the peak of infection must be $\approx 3$ to 30 times the observed peak viral load.

\section{Calculating the Total Number of Cells Infected with SARS-CoV-2}

We use our estimate of the total number of infectious units in the body of an infected individual to estimate the number of cells that are infected by the virus during peak infection. In order to estimate the total number of infected cells, we estimate how many infectious units are found in each infected cell as shown in Fig. 2.

We rely on two lines of evidence in order to estimate the number of infectious units within an infected cell at a given time. The first is data regarding the total number of infectious units produced by an infected cell throughout its lifetime, also known as the yield. As we are not aware of studies directly reporting values of the yield of cells infected with SARS-CoV-2, we used values reported for other betacoronaviruses in combination with values we derived from a study (21) of replication kinetics of SARS-CoV-2. Using a plaque formation assay to count the number of infectious units, two previous studies measured the viral yield as either 10 to 100 or 600 to 700 infectious units (22, 23). Using reported values for replication kinetics of SARS-CoV-2 (21), we estimated a yield of $\sim 10$ infectious units per cell at $36 \mathrm{~h}$ to $48 \mathrm{~h}$ after infection, in agreement with the lower end of these estimates. To convert the total number of infectious units produced overall by a cell into the number of units residing in the cell at a given moment, we estimate the ratio between these two quantities to be 3 to 30 , using two independent methods detailed in SI Appendix. Combining this ratio with our estimate for the total number of units produced by a cell, we thus estimate that, at any given moment, there are somewhere between a few and a few hundred infectious units residing in each infected cell.

The second line of evidence concerns the density of virions within a single cell. Several studies have used transmission electron microscopy (TEM) to characterize the intracellular replication of SARS-CoV-2 virions within cells (24-27). Using seven TEM scans taken from those studies, we estimated that the density of virions within infected cells is $10^{5}$ virions per $1 \mathrm{pL}$ (Dataset $\mathrm{S} 1$ ). As the human cells targeted by SARS-CoV-2 have a volume of $\approx 1 \mathrm{pL}$ (resulting in a cellular mass of $\approx 1 \mathrm{ng}$ ) $(28,29)$, TEM data indicate there are $\approx 10^{5}$ viral particles within a single infected cell at any point in time. As done above, we assume a ratio of one infectious unit resulting per $10^{4}$ virions. Thus, TEM scans imply that there are $\approx 10$ infectious units that will result from the virions residing inside a cell at any given moment after the initial stages of infection.

Following those lines of evidence, we conclude that, at a given moment, there are $\sim 10^{5}$ virions residing inside an infected cell, which translates into $\sim 10$ infectious units. Using the ratio of total production to the value at a given time inside the cell, we further conclude that the overall yield from an infected cell is $\sim 10^{5}$ to $10^{6}$ virions or $\sim 10$ to 100 infectious units, coinciding with the middle range of measurements from other betacoronaviruses. This estimate also agrees well with recent results from dynamical models of SARS-CoV-2 host infection $(30,31)$.

We can perform a sanity check using mass considerations to see that our estimate of the number of virions is not beyond the maximal feasible amount. Each virion has a mass of $\approx 1 \mathrm{fg}(5)$. Hence, $10^{5}$ virions have a mass of $\approx 0.1 \mathrm{ng}$, about $10 \%$ of the total mass of a 1-ng host cell and about a third of its dry weight. While a relatively high fraction, this is still within the range observed for other viral infections $(32,33)$.

Combining the estimates for the overall number of infectious units in a person near peak infection and the number of infectious units in a single cell $\left(C_{\text {infectious units per cell }}\right)$, we can calculate the number of infected cells around peak infection,

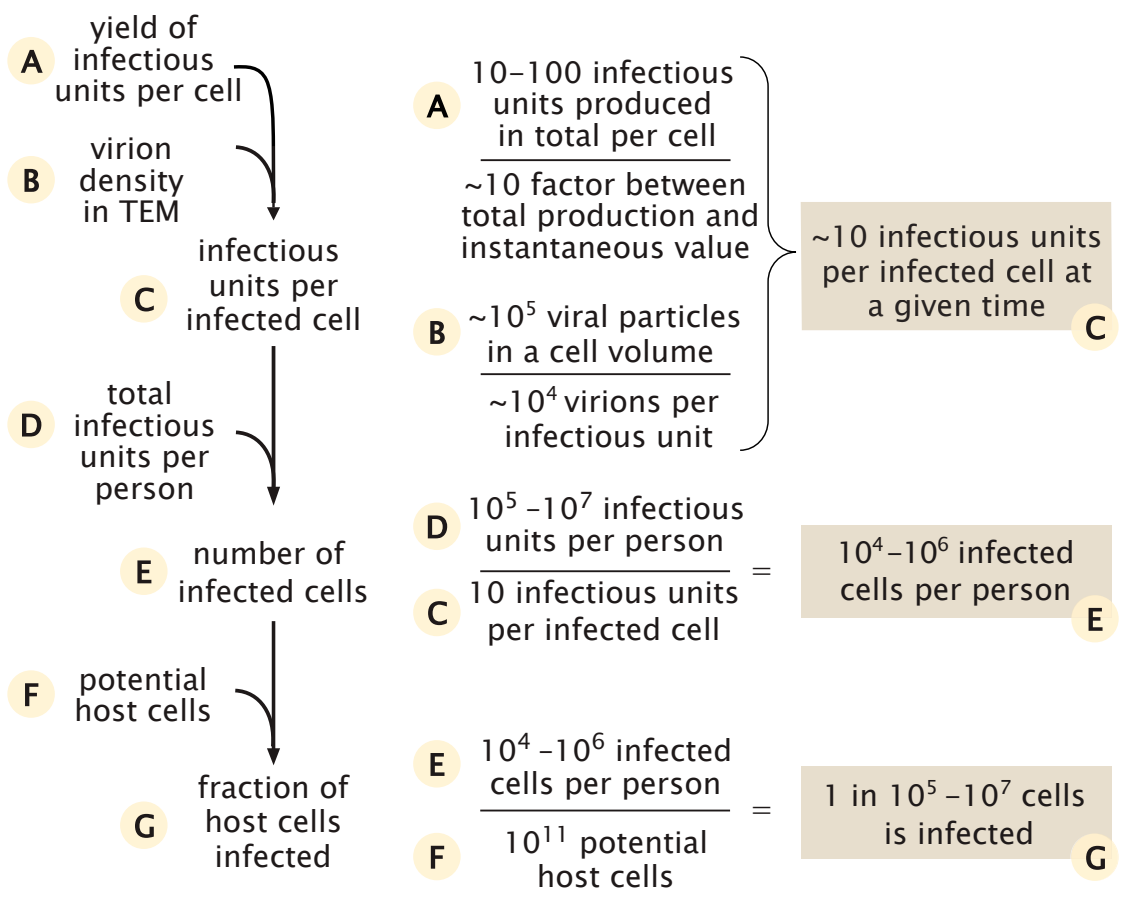

Fig. 2. Estimate of the number of infected cells and their fraction out of the potential relevant host cells. 


$$
\begin{gathered}
\frac{N_{\text {infectious units }}}{C_{\text {infectious units per cell }}}=N_{\text {infected cells }} \\
\frac{10^{5} \text { to } 10^{7} \text { [infectious units] }}{10 \text { [infectious units per cell] }}=10^{4} \text { to } 10^{6} \text { [cells]. }
\end{gathered}
$$

How does this estimate compare to the number of potential host cells for the virus? The best-characterized route of infection for SARS-CoV-2 is through cells of the respiratory system, specifically, the pneumocytes $\left(\sim 10^{11}\right.$ cells $)$, alveolar macrophages $\left(\sim 10^{10}\right.$ cells), and the mucus cells in the nasal cavity $\left(\sim 10^{9}\right.$ cells $)(28,29)$. Other cell types, like enterocytes (gut epithelial cells), can also be infected (34), but they represent a similar number of cells (35) and therefore don't change the order of magnitude of the potential host cells. As such, our best estimate for the size of the pool of cell types that SARS-CoV-2 likely infects is thus $\sim 10^{11}$ cells, and the number of cells infected during peak infection therefore represents a small fraction of this potential pool (one in $10^{5}$ to $10^{7}$ ).

\section{Discussion}

Our quantitative analysis establishes estimates for the absolute number of virions present in an infected individual, as well as the number of virions produced during the infection and the total number of infected cells in the body. There are various ways in which one can leverage such quantitative estimates to produce insights regarding COVID-19. First, having absolute estimates allows us to compare them to other quantities in the human body and thus put the number of virions in context and arrive at meaningful insights. For example, a human body comprises $\approx 3 \times$ $10^{13}$ cells (3). This means that, even for our highest estimate, i.e., $10^{11}$ virions per host, human cells outnumber the virions by more than 100-fold. We can also compare our estimate for the total number of infected cells with the total pool of cells expressing ACE2 (angiotensin-converting enzyme 2) and TMPRSS2 (transmembrane protease, serine 2), the receptor and main protease SARS-CoV-2 relies on for infecting cells. Single-cell RNAsequencing studies (36-38) indicate that a few percent of the cells in the lungs and airways express ACE2 and TMPRSS2. Most of the cells that have been found to express both are type 2 pneumocytes. While these results might be biased due to dropout effects in measurements of only a few molecules $(38,39)$, it is still reasonable that 1 to $10 \%$ of the lung and airway cells contain the necessary receptor to be infected by SARS-CoV-2, totaling $\sim 10^{9}$ cells. This number is several orders of magnitude higher than our estimate for the total number of infected cells during peak infection $\left(10^{4}\right.$ to $\left.10^{6}\right)$. This suggests that, out of the cells expressing both ACE2 and TMPRSS2, only a small fraction, e.g., $10^{-5}$ to $10^{-3}$, are infected by the virus.

Because the immune system is the main line of defense against SARS-CoV-2, it is interesting to quantitatively examine the known immune response in comparison with the viral loads we estimated here. For example, we can compare the peak number of viral particles $\left(10^{9}\right.$ to $\left.10^{11}\right)$ to the number of antibodies the body produces to combat SARS-CoV-2 infection. Levels of SARS-CoV-2-specific IgG antibodies $\left(C_{I g G}\right)$ were measured 3 wk after the onset of symptoms, showing a serum concentration of $\sim 10 \mu \mathrm{g} / \mathrm{mL}(40)$. Only $\approx 5 \%$ of the total anti-spike (the viral protein responsible for allowing the attachment and fusion with the host cell) IgG antibodies has the capacity to neutralize the virus $\left(f_{\text {neutralizing }}\right)(41)$. Combining the concentration of neutralizing $\mathrm{IgG}$ antibodies with a mean $\mathrm{IgG}$ molecular weight $\left(M W_{I g G}\right)$ of $150 \mathrm{kDa}(42)$, we estimate the number of neutralizing antibodies per $\mathrm{mL}$ of serum $\left(C_{\text {neutralizing }}\right)$,

$$
C_{I g G} \times f_{\text {neutralizing }} \times \frac{1}{M W_{I g G}} \times N_{\text {Avogadro }}=C_{\text {neutralizing }}
$$

$$
\begin{aligned}
& 10^{-5}[\mathrm{~g} \text { IgG per } \mathrm{mL}] \times 5 \%[\text { neutralizing per } \mathrm{IgG}] \\
& \times \frac{1}{150,000[\mathrm{~mol} \text { per } \mathrm{g}] \times 6 \times 10^{23}[\text { molecules per mol }]} \\
& \quad=3 \times 10^{12}[\text { neutralizing molecules per } \mathrm{mL}]
\end{aligned}
$$

Combining this estimate with the measurement of viral concentration within the lung tissue and accounting for 30 to 40 spike trimers on each SARS-CoV-2 virion $(43,44)$, we can estimate the ratio of neutralizing antibodies to viral spike proteins as

$$
\frac{C_{\text {neutralizing }}}{C_{\text {virions }}^{\text {lungs }} \times N_{\text {spike proteins }}}=R_{\text {neutralizing antibodies } / \text { spike proteins }}
$$

$$
\begin{aligned}
& \frac{3 \times 10^{12}[\text { neutralizing molecules per } \mathrm{mL}]}{\left.10^{6} \text { to } 10^{8} \text { [viral particles per } \mathrm{mL}\right] \times 30} \\
& =10^{3} \text { to } 10^{5} \text { [neutralizing antibodies per spike protein]. }
\end{aligned}
$$

Previous work on other morphologically similar RNA viruses like influenza and flavivirus found that a ratio of one bound neutralizing antibody per two to four receptor-binding proteins was sufficient to neutralize binding of a virion to its cellular receptor in vitro $(45,46)$. Taken at face value, our estimate seems to suggest an excess of neutralizing antibodies. There are several factors that will cause the effective concentration of antibodies the virus experiences to be lower. First, the antibody concentrations in the lung tissue tend to be lower than that of the blood. Second, many of the spike proteins are extensively glycosylated. These glycosylations shield many of the binding sites for neutralizing antibodies (44) and thus decrease the efficiency of neutralization (47). However, it is important to remember that the most relevant measure for the effectiveness of antibody neutralization is the fraction of viral spike proteins that are bound by neutralizing antibodies. This fraction is determined by the strength of the binding of the neutralizing antibodies $(\mathrm{nAb})$ to the viral particles, given by the dissociation constant $K_{d}$ (46). Following the first-order relation,

$$
\text { fraction bound by } \mathrm{nAb}=\frac{[\mathrm{nAb}]}{[\mathrm{nAb}]+K_{d}} \text {. }
$$

As the dissociation constants for antibody-epitope binding are mostly in the range of $1 \mathrm{~nm}$ to $10 \mathrm{nM}(48,49)$, we get

$$
\begin{aligned}
{[\mathrm{nAb}]=} & 3 \times 10^{12}[\text { neutralizing molecules per } \mathrm{mL}] \\
& \times \frac{1}{6 \times 10^{23}}[\mathrm{~mol} \text { per molecule }] \times 1,000[\mathrm{~mL} / \mathrm{L}] \\
= & 3 \times 10^{-9}[\mathrm{~mol} / \mathrm{L}]=3[\mathrm{nM}]
\end{aligned}
$$

$$
\text { fraction receptors bound by } \mathrm{nAb}=\frac{3[\mathrm{nM}]}{3[\mathrm{nM}]+1 \text { to } 10[\mathrm{nM}]}
$$$$
=25 \text { to } 75 \% \text {. }
$$

Thus, even though the ratio between the number of neutralizing antibodies and viral particles is high, such a high number of antibodies is essential to ensure that enough of the epitopes are bound (an even higher ratio is needed for some antiviral drugs, as shown in SI Appendix).

Beyond the humoral arm of the immune response, $\mathrm{T}$ cells are also an integral part of the targeting of viral antigens. Although 
severe cases of COVID-19 tend to have lower concentrations of $\mathrm{T}$ cells in the blood, they have a higher fraction of SARSCoV-2-specific $\mathrm{T}$ cells than mild COVID-19 cases (50). Here SARS-CoV-2-specific T cells denotes T cells that showed markers for activation and proliferation after stimulation with SARSCoV-2 peptide pools (50). We can use the concentrations of CD4+ and CD8+ cells in the blood in combination with their fraction of SARS-CoV-2-specific cells (50) to estimate one to two CD4+ cells per $\mu \mathrm{L}$ and 0.2 to $0.3 \mathrm{CD} 8+$ cells per $\mu \mathrm{L}$ specific for SARS-CoV-2 in convalescent patients and severe cases. Assuming a patient's blood volume is $\sim 5 \mathrm{~L}$ and that 1 to $2 \%$ of lymphocytes reside in the blood (35), we estimate that there are up to $10^{9}$ SARS-CoV-2-specific T cells in severe cases, with an unknown fraction found in the infected tissue, or one per 1 to 100 viral particles at the peak of infection, and $10^{2}$ to $10^{4}$ such $\mathrm{T}$ cells per infected cell.

In our comparisons, we usually rely on our estimates for the characteristic values for the peak viral load in infected individuals, which correspond to the center of the distribution of the measured values (specifically, the interquartile range-between the quantiles $25 \%$ and $75 \%$ ). However, it is important to note that there is a high degree of variability in viral loads, exceeding six orders of magnitude, as can be seen from samples taken from the upper respiratory system (51). This wide variation reflects the difference between people as well as differences in viral load through the progression of infection within an infected individual (52). Thus, extreme cases could exceed the interquartile range provided by an additional two orders of magnitude, reaching values of $10^{13}$ viral particles in a single person at the peak of infection, while up to $10 \%$ of the cells expressing both ACE2 and TMPRSS2 are infected. The variation in the number of virions, as related to the severity of the disease and its outcome, is detailed in SI Appendix. It is also important to note that viral load in different tissues in the host body changes throughout the infection, with some tissues likely infected early on and others later in the infection (53)

Another way in which we can use our estimates to produce insights is by taking a global view and extrapolating from the numbers observed in a single infected individual to the entire population. For example, we can estimate the number of viral particles residing in all infected humans at a given time. The total number of viral particles at peak infection was shown above to be $10^{9}$ to $10^{11}$ viral particles (this range corresponds to the 25 th to 75 the percentile range). Because the viral loads of individuals are roughly log-normally distributed (17), the arithmetic average of the number of viral particles at peak infection would be on the high end of the range, even beyond the 75 th percentile $\left(10^{11}\right.$ to $10^{12}$ particles). There is a rapid drop in viral loads after peak infection; thus the total number of viral particles is dominated by those infected individuals who are close to the infection peak (within $1 \mathrm{~d}$ to $2 \mathrm{~d}$ ). Assuming that, during most of the course of the pandemic, there has been a total of 1 million to 10 million infected people close to peak infection globally at any given time (including those undetected; see SI Appendix for details) (54), we arrive at a total of $10^{17}$ to $10^{19}$ viral particles or $10^{13}$ to $10^{15}$ infectious units at any given time. Similarly, the arithmetic mean of the number of particles produced over the course of infection of an average individual is $10^{12}$ to $3 \times 10^{13}$ viral particles ( $\bar{N}_{\text {viral particles produced per person }}$ ), or $10^{8}$ to $3 \times 10^{9}$ infectious units (see SI Appendix for the detailed derivation of the uncertainty range).

One can contextualize these estimates using an absolute mass perspective. Each virion has a mass of $\approx 1 \mathrm{fg}(5)$. Therefore, even when the body carries $10^{9}$ to $10^{11}$ viral particles, these have a mass of only about $1 \mu \mathrm{g}$ to $100 \mu \mathrm{g}$, that is, 1 to 100 times less than the mass of a poppy seed. The total mass of virions residing in humanity at a given time is on the order of $0.1 \mathrm{~kg}$ to $10 \mathrm{~kg}$. Furthermore, using the total number of viral particles produced throughout an infection, we can derive the total mass of all the SARS-CoV-2 viral particles ever produced throughout this current pandemic (concentrating on humans, which we find to currently dominate over animal reservoirs). We assume the total number of infected people will be in the range of 0.5 billion to 5 billion people, representing optimistic and pessimistic future scenarios for the pandemic (see SI Appendix for details). To calculate the total number of virions that will have been produced by the end of the pandemic, we multiply the total number of infected people by the total number of viral particles produced over an infection of an average person (which is the arithmetic mean of the distribution across people). We then multiply this number by the average mass of a single virion to find the total mass of viral particles produced globally for such widespread infection (see SI Appendix for details of the uncertainty estimate),

$$
\begin{aligned}
& N_{\text {infected people }} \times M_{\text {viral particle }} \times \bar{N}_{\text {viral particles produced per person }} \\
& =M_{\text {all viral particles produced }}
\end{aligned}
$$

$$
\begin{aligned}
& (0.5 \text { to } 5) \times 10^{9} \text { [people] } \times 10^{-18}[\mathrm{~kg} \text { per viral particle] } \\
& \times\left(10^{12} \text { to } 3 \times 10^{13}\right) \text { [viral particles produced per person] } \\
& =10^{3} \text { to } 10^{5}[\mathrm{~kg}]
\end{aligned}
$$

Finally, we use our estimates of the total number of viruses in an infected human to examine the evolution of SARS-CoV-2 and, specifically, estimate the rate of emergence of new variants. When studying the genetic diversity of SARS-CoV-2, we can define two different measures for diversity. The first is the diversity along a genetic lineage of virions-propagating from the ancestral strain in Wuhan until currently circulating virions. The second is the diversity among a population of virions-for example, the population of virions present in the body of an infected individual. We start by calculating the average number of mutations accumulated along a specific lineage of ancestor virions leading from the beginning of viral replication in the host until the end of host infection. In these calculations, we rely on estimates of the mutation rate per replication cycle per site $(3 \times$ $10^{-6} \mathrm{nt}^{-1}$ per cycle) which have been measured for MHV, another betacoronavirus (5). We further assume that each human host is infected by a few infectious units (55-57), and use the estimated yield of $\sim 10$ to 100 infectious units per cell. Each cycle of infection is therefore assumed to produce 10 to 100 infectious units that, in turn, go on to infect other cells. As estimated above, there are $3 \times 10^{5}$ to $3 \times 10^{8}$ infectious units produced over the course of an infection. Assuming exponential growth, the entire course of infection will therefore take three to seven viral replication cycles (Fig. $3 A$ ). As the SARS-CoV-2 genome has a length of 30,000 nucleotides (nts), we can compute the expected number of mutations accumulating in a virus that is the product of three to seven replication cycles using the per cycle mutation rate,

$$
\begin{aligned}
& 3 \times 10^{4}[\mathrm{nt}] \times 3 \text { to } 7 \text { [cycles per infection] } \times 3 \\
& \times 10^{-6}[\text { mutations per nt per cycle] } \\
& \approx 0.5[\text { mutations per infection] }
\end{aligned}
$$

Therefore, if we track a single lineage of virions from the time they started replicating in the body until the end of the infection, this lineage would accumulate in the range of 0.1 to 1 mutations on average across its entire genome (Fig. $3 A$ ). Considering that the mean time between successive infections, known as the generation interval, is about $4 \mathrm{~d}$ to $5 \mathrm{~d}$, we can estimate an overall rate of $\approx 3$ mutations per month over the course of the epidemic 
(Fig. 3B). This is consistent with empirical values observed during the pandemic for SARS-CoV-2 of about $10^{-3} \mathrm{nt}^{-1} \cdot \mathrm{yr}^{-1}(58,59)$, also known as the evolution rate. The evolution rate is estimated from the observed rate of mutation accumulation across sequenced genomes from different time points over the course of the pandemic using reconstruction of phylogenetic trees (59). It therefore includes both the rate of accumulation of neutral mutations and the effects of natural selection. This estimated rate of evolution

\section{A IN-HOST EVOLUTION}

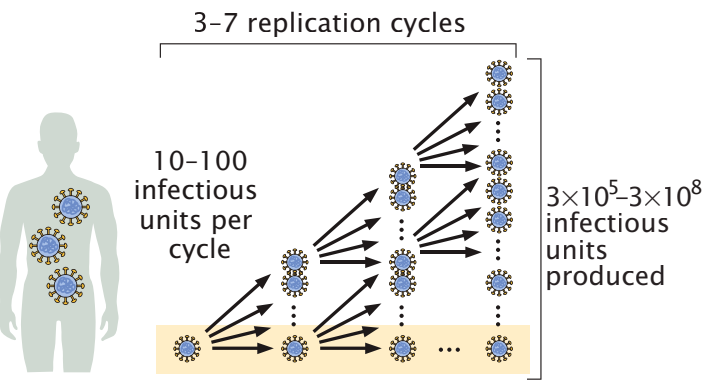

total genetic variation

\begin{tabular}{|c|c|c|c|c|}
\hline $\begin{array}{c}3 \times 10^{5}-3 \times 10^{8} \\
\text { infectious } \\
\text { units } \\
\text { produced }\end{array}$ & $x$ & $\begin{array}{c}0.5 \\
\text { mutations } \\
\text { per lineage }\end{array}$ & $\approx$ & $\begin{array}{c}10^{5}-10^{8} \\
\text { mutations } \\
\text { per host }\end{array}$ \\
\hline
\end{tabular}

$\left.\begin{array}{c}3 \times 10^{4} \\ \text { bases }\end{array} \times \begin{array}{c}3 \text { alternative } \\ \text { bases }\end{array}\right]\left[\begin{array}{c}10^{5} \\ \text { single bases } \\ \left(10^{5}\right)^{2}=10^{10} \\ \text { two bases } \\ \text { combinations }\end{array}\right.$ variation spans most single base mutations

at most $1 \%$ of the two bases combinations

mutation rate of a specific lineage

\begin{tabular}{|c|c|c|c|c|c|c|}
\hline $\begin{array}{l}\quad 3-7 \\
\text { replication } \\
\text { cycles per } \\
\text { infection }\end{array}$ & $x$ & $\begin{array}{c}3 \times 10^{-6} \\
\text { mutations } \\
\text { per nt per } \\
\text { cycle }\end{array}$ & $x$ & $\begin{array}{c}3 \times 10^{4} \\
\text { nucleotides }\end{array}$ & $\approx$ & $\begin{array}{c}0.5 \\
\text { mutations } \\
\text { per lineage } \\
\text { per infection }\end{array}$ \\
\hline
\end{tabular}

\section{B \\ INTER-HOST EVOLUTION}

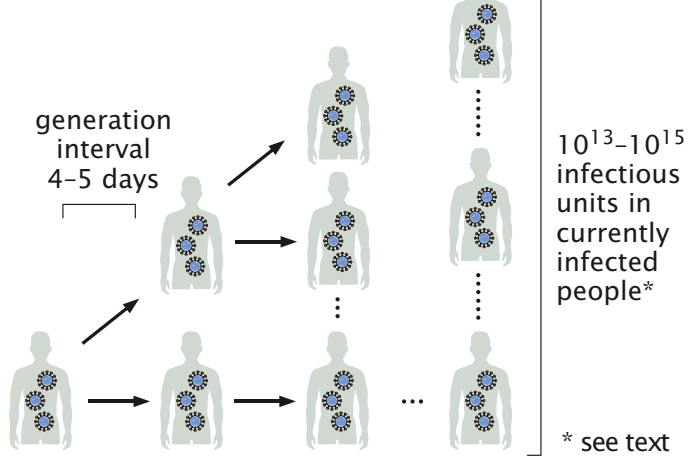

total genetic variation

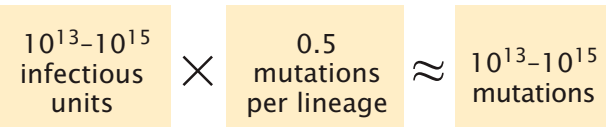

possible mutations

$$
\left(10^{5}\right)^{2}=10^{10}
$$

two bases

$\begin{gathered}3 \times 10^{4} \\ \text { bases }\end{gathered} \times \begin{gathered}3 \text { alternative } \\ \text { bases }\end{gathered}\left[\begin{array}{l}\text { combinations } \\ \left(10^{5}\right)^{3}=10^{15} \\ \text { three bases }\end{array}\right.$ combinations

\section{variation spans all possible two base combinations \\ variation does not span all possible three base combinations}

\begin{tabular}{|c|c|c|c|c|}
\hline $\begin{array}{c}0.5 \\
\text { mutations } \\
\text { per } \\
\text { infection }\end{array}$ & $\div$ & $\begin{array}{c}\text { 4-5 } \\
\text { days } \\
\text { between } \\
\text { infections }\end{array}$ & $\approx$ & $\begin{array}{c}3 \text { mutations } \\
\text { per month; } \\
10^{-3} \text { mutations } \\
\text { per nucleotide per year }\end{array}$ \\
\hline
\end{tabular}

evolution rate

\begin{tabular}{|c|c|c|c|c|}
\hline $\begin{array}{c}0.5 \\
\text { mutations } \\
\text { per } \\
\text { infection }\end{array}$ & $x$ & $\begin{array}{c}0.3-3 \times 10^{6} \\
\text { world wide } \\
\text { infections } \\
\text { per day }\end{array}$ & $\approx$ & $\begin{array}{c}0.1-1 \times 10^{6} \\
\text { mutations transmitted } \\
\text { per day world wide }\end{array}$ \\
\hline $\begin{array}{l}3 \times 10^{4} \\
\text { bases }\end{array}$ & $x$ & $\begin{array}{c}3 \\
\text { alternative } \\
\text { bases }\end{array}$ & $\approx$ & $\begin{array}{l}10^{5} \\
\text { possible single } \\
\text { base mutations }\end{array}$ \\
\hline
\end{tabular}

\section{WORLD WIDE MUTATION POTENTIAL}

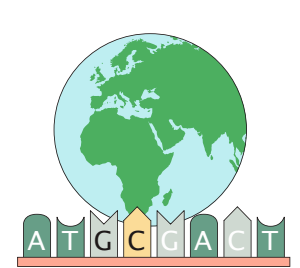

every single base mutation is being generated de-novo and transmitted to a new host every day

Fig. 3. The relationship between the number of virions produced in an infected individual and the evolution of SARS-CoV-2. We use our estimates for the total number of virions produced during an infection, along with other epidemiological and biochemical characteristics of SARS-CoV-2, to estimate the rate of mutation accumulation within an infected host $(A)$ and within the population $(B)$. We consider both the evolution along a specific genetic lineage of virions and the diversity among a population of virions-either within an infected host $(A)$ or within the total population $(B)$. In addition, we look at the de novo mutation generated and transmitted to the newly infected in comparison to all possible single base mutations (C). 
matches the number of mutations observed in variants present today, about a year after the onset of the pandemic, most of which contain about 20 to 30 mutations. The extreme examples in terms of number of mutations, of variants such as B.1.1.7, accumulated closer to 40 mutations compared to the first strains isolated.

We can use our estimates of the viral mutation rate to assess the expected rate of appearance of a specific single base mutation. Consider the example of a single nucleotide substitution resulting in the E484K mutation in which the Glutamate $(E)$ in position 484 is replaced with Lysine $(\mathrm{K})$. This mutation requires a specific substitution in a specific location: The first base of the codon must change from $\mathrm{G}$ to $\mathrm{A}$. As each nucleotide can mutate to three others (e.g., G can become A, T, or C) and the genome contains 30,000 nucleotides, there are $\approx 100,000$ possible single nucleotide substitutions to the SARS-CoV-2 genome. As concluded above, about 0.5 mutations are accumulated in every host infection cycle. Without accounting for the effects of selection (i.e., assuming the mutant virions are equally capable of infection and propagation), or the varying chances of mutation among nucleotides, we expect that such a specific mutation will be observed in one out of every $\sim 200,000$ infections. Over recent months, hundreds of thousands of cases have been identified across the world every day, and many additional cases have likely gone unidentified. Indeed, as shown in Fig. $3 C$, the estimated number of mutations generated daily $\left(10^{5}\right.$ to $10^{6}$ mutations per day) likely exceeds the total number of possible single nucleotide substitutions to the SARS-CoV-2 genome $\left(\approx 10^{5}\right.$ substitutions $)$ assuming 0.3 million to 3 million new cases a day worldwide. As such, our estimates imply that every single base mutation is being generated de novo and transmitted to a new SARS-CoV-2 host, somewhere in the world, every day.

In addition to considering a specific lineage of SARS-CoV-2 viruses, we can also consider the genetic diversity at the population level and estimate the total variability across the entire repertoire of infectious units produced during a single course of infection. As we estimated that $3 \times 10^{5}$ to $3 \times 10^{8}$ infectious units are produced during an infection, each one resulting from a lineage of ancestors and mutations, we expect, overall, to have about $10^{5}$ to $10^{8}$ mutations across all of the infectious units. Some of these mutations that occurred in early cycles will appear in many later progeny within the host, while those generated in the most recent cycle will appear in only one viral genome. Because the SARS-CoV-2 genome is 30,000 nucleotides long, the $10^{5}$ to $10^{8}$ mutations across all of the virions produced over the course of a single infection probably cover every possible single nucleotide substitution (Fig. $3 A$ ). They even cover a significant fraction of the possible pairs of single nucleotide substitutions. If we look globally at the entire number of infectious units of SARS-CoV-2 currently present within the infected human population, which we estimated above at $10^{13}$ to $10^{15}$, we expect that every combination of two nucleotide substitutions and many, though not all, three nucleotide substitutions will be present in at least one infectious unit (Fig. 3B).

This large genetic diversity might naively imply that advantageous mutations will rapidly take over the population due to natural selection, but there are several factors which slow down the rate of selection. These factors include epistasis, a phenomenon where a single mutation becomes advantageous only when other specific mutations occurred previously. Another key factor is the genetic bottleneck imposed during the transmission of virions between infected individuals. These bottlenecks are expected to slow selection, as only a tiny fraction of the diversity generated in the host is passed on to future generations (55-57). This quantitative understanding brings into focus cases in which selection can occur for a significant amount of time with no bottlenecks, such as the case of long and persistent infections, for example, in immunocompromised patients $(60-62)$. We thus conclude that careful accounting of the number of virions can give insight into the process of viral evolution within and across hosts.

One of the strengths of a holistic quantitative analysis such as the one performed here is its ability to expose interesting "quirks" that are otherwise elusive. One such observation is the ratio of $\sim 10^{4}$ between the RNA copies measured using RT-PCR and the number of infectious units measured in TCID $_{50}$. Ratios on the order of $10^{3}$ to $10^{4}$ between viral particles and PFUs were observed in animal viruses such as poliovirus and papillomavirus (63). Naively, such a ratio would suggest that only $0.01 \%$ of the virions produced are actually infectious. This ratio implies that SARS-CoV-2 is not very efficient in producing infectious progeny. While we do not have a clear explanation for this seeming low efficiency, there are several possible factors that will affect this ratio. First, measuring RNA copies may not correspond directly to actual virions but also measures naked viral RNA. Second, while TCID $_{50}$ is the most widely available assay for measuring infectious titer, it may not accurately reflect the actual number of infectious virions, for example, because conditions in the assay may not be optimal for SARS-CoV-2 infection. Another possibility is that many virions are noninfectious due to the neutralizing effect of binding antibodies, and thus the ratio may represent the effect of the immune response, and change over the period of infection.

Beyond exposing these quantitative aspects, a holistic analysis allows us to identify major knowledge gaps in the available literature. For example, the virion yield per infected cell is known only from a few studies on different kinds of betacoronavirus from over 40 y ago $(22,23)$. Similarly, measurements of the mutation rate per nucleotide per cycle in SARS-CoV-2 are of much interest but missing. As discussed above, the quantitative relationship between viral RNA copies, viral particles, and infectious units is not fully characterized for SARS-CoV-2, and thus further research could help better constrain and explain the differing values. In addition, a model describing the quantitative relationship between antibody production and infection metrics would help quantitatively test the estimates presented here.

Establishing estimates for the total number and mass of SARS-CoV-2 virions in infected individuals allows us to connect together various aspects of the pandemic, from immunology to evolution, and to highlight emerging patterns and relationships not obviously evident. Having better quantitative information on the process of infection at the cellular level, the intrahost level, and the interhost level will hopefully empower researchers with better tools to combat the spread of COVID-19 and to understand its evolution, including the rise of variants of concern.

\section{Materials and Methods}

The derivation of the main results of the study are presented in the Estimate of the Number of Virions in an Infected Individual section. Here we describe essential methods not discussed in detail elsewhere in the text. Additional information can be found in SI Appendix.

Number and Fraction of Infected Cells. The total number of infected cells was estimated by dividing the peak number of virions within an infected human by the instantaneous number of virions residing in a cell. The instantaneous number of virions in an infected cell was estimated by two methods: 1) using the total yield of virions from an infected cell and 2) using an estimate of the density of viral particles within infected cells. In the first method, we start with the per-cell viral yield (10 to 100 infectious units), and convert it into an instantaneous number of virions using a conversion factor of 3 to 30 . This conversion factor equals the ratio of total production of virions to the peak viral load, which we derive in the Estimate of the Number of Virions in an Infected Individual section and SI Appendix. In the second method, estimates for the density of viral particles within cells were derived by two independent viewers counting viral particles in TEM images from the literature (24-27). Counts were converted to densities by dividing the total particle counts by the volume of the slice captured by the image, which was estimated as the area covered by the image multiplied by the diameter of a 
virion. The fraction of susceptible cells that are infected with SARS-CoV-2 was calculated by comparison to literature values for the number of cells in the airway system as detailed in the Calculating the Total Number of Cells Infected with SARS-CoV-2 section $(28,29)$.

Number of Virions within an Average Infected Person and within the Entire Infected Population. We estimated the number of virions in an average infected person as the arithmetic average of the distribution of total viral load across individuals. We assumed the viral loads are distributed log-normally. We assumed the coefficient of variation of the distribution is similar to that of the distribution of the peak viral load found in ref. 17. The number of virions within currently infected humans was then estimated by multiplying this arithmetic average by the number of humans near peak infection. The number of humans near peak infection was chosen to represent the typical number of daily new cases reported in online tracking websites (54) multiplied by $1 \mathrm{~d}$ to $3 \mathrm{~d}$ to account for the characteristic time an infected individual spends at near-peak viral load. Similarly, the total number of virions produced over the pandemic was estimated using probable scenarios for the total number of cases multiplied by the arithmetic average of the total production of virions over a single infection (see SI Appendix for details). The total mass of virions was then derived by multiplying with the average mass of a single virion. See SI Appendix for uncertainty estimation.

Mutation Rate. To estimate the number of mutations occurring during a single infection, we relied on previous estimates of the molecular mutation rate (5) and the number of replication cycles following Eq. 8. The number of replication cycles within an individual was estimated assuming exponential

1. U. Moran, R. Phillips, R. Milo, SnapShot: Key numbers in biology. Cell 141, 1262-1262.e1 (2010).

2. R. Sender, S. Fuchs, R. Milo, Are we really vastly outnumbered? Revisiting the ratio of bacterial to host cells in humans. Cell 164, 337-340 (2016).

3. R. Sender, S. Fuchs, R. Milo, Revised estimates for the number of human and bacteria cells in the body. PLoS Biol. 14, e1002533 (2016).

4. Y. M. Bar-On, R. Phillips, R. Milo, The biomass distribution on Earth. Proc. Natl. Acad. Sci. U.S.A. 115, 6506-6511 (2018).

5. Y. M. Bar-On, A. Flamholz, R. Phillips, R. Milo, SARS-CoV-2 (COVID-19) by the numbers elife 9, e57309 (2020).

6. J. B. Case, A. L. Bailey, A. S. Kim, R. E. Chen, M. S. Diamond, Growth, detection quantification, and inactivation of SARS-CoV-2. Virology 548, 39-48 (2020).

7. L. J. Reed, H. Muench, A simple method of estimating fifty percent endpoints. Am J. Epidemiol. 27, 493-497 (1938).

8. V. J. Munster et al., Respiratory disease in rhesus macaques inoculated with SARS-CoV-2. Nature 585, 268-272 (2020).

9. A. Chandrashekar et al., SARS-CoV-2 infection protects against rechallenge in rhesus macaques. Science 369, 812-817 (2020).

10. C. Shan et al., Infection with novel coronavirus (SARS-CoV-2) causes pneumonia in Rhesus macaques. Cell Res. 30, 670-677 (2020)

11. W. S. Snyder et al., Report of the Task Group on Reference Man (ICRP Publ. 23, Pergamon, 1975)

12. International Commission on Radiological Protection, Basic anatomical and physiological data for use in radiological protection reference values. Ann. ICRP 32, 5-265 (2002)

13. B. Rockx et al., Comparative pathogenesis of COVID-19, MERS, and SARS in a nonhuman primate model. Science 368, 1012-1015 (2020).

14. H. Salje et al., Estimating the burden of SARS-CoV-2 in France. Science 369, 208-211 (2020).

15. World Health Organization, "Report of the WHO-China Joint Mission on Coronavirus Disease 2019 (COVID-19)" (World Health Organization, 2020).

16. T. C. Jones et al., An analysis of SARS-CoV-2 viral load by patient age. medRxiv [Preprint] (2020). https://doi.org/10.1101/2020.06.08.20125484 (Accessed 9 September 2020).

17. S. M. Kissler et al., SARS-CoV-2 viral dynamics in acute infections. bioRxiv [Preprint] (2020). https://doi.org/10.1101/2020.10.21.20217042 (Accessed 31 March 2021).

18. P. Z. Chen et al., Heterogeneity in transmissibility and shedding SARS-CoV-2 via droplets and aerosols. medRxiv [Preprint] (2020). https://doi.org/10.1101/2020.10.13. 20212233 (Accessed 31 March 2021).

19. S. Wang et al., Modeling the viral dynamics of SARS-CoV-2 infection. Math. Biosci. 328, 108438 (2020).

20. K. Hattaf, N. Yousfi, Dynamics of SARS-CoV-2 infection model with two modes of transmission and immune response. Math. Biosci. Eng. 17, 5326-5340 (2020).

21. J. A. Plante et al., Spike mutation D614G alters SARS-CoV-2 fitness. Nature 592, 116-121 (2021)

22. J. A. Robb, C. W. Bond, Pathogenic murine coronaviruses. I. Characterization of biological behavior in vitro and virus-specific intracellular RNA of strongly neurotropic JHMV and weakly neurotropic A59V viruses. Virology 94, 352-370 (1979).

23. N. Hirano, K. Fujiwara, M. Matumoto, Mouse hepatitis virus (MHV-2). Plaque assay and propagation in mouse cell line DBT cells. Jpn. J. Microbiol. 20, 219-225 (1976).

24. M. Imai et al., Syrian hamsters as a small animal model for SARS-CoV-2 infection and countermeasure development. Proc. Natl. Acad. Sci. U.S.A. 117, 16587-16595 (2020). growth from one infectious unit to the total number of infectious units produced within an infected individual, $3 \times 10^{5}$ to $3 \times 10^{8}$. Based on our estimates of the per-cell yield of infectious units, a factor of 10 to $100 \mathrm{in}$ fectious units was used per viral replication cycle. The total evolution rate was derived from the mutation rate by dividing the total number of mutations during a single infection by the generation interval. The genetic variation in the viral population within an individual (or the entire human population) was estimated from the mutation rate by multiplication by the number of infectious units produced over a single infection (or the total number of infectious units within the currently infected population).

Data Availability. All study data are included in the article, SI Appendix, and Dataset S1.

ACKNOWLEDGMENTS. We thank Itai Benhar, Gidon Eshel, Shai Fuchs, Thierry Mora, Eran Segal, Maya Shamir, Ziv Shulman, Huicheng Shi, Harinder Singh, Einat Vitner, Aleksandra Walczak, and John Yin for valuable feedback on this manuscript. This research was supported by the European Research Council (Project NOVCARBFIX 646827), Israel Science Foundation (Grant 740/ 16), Beck-Canadian Center for Alternative Energy Research, Dana and Yossie Hollander, Ullmann Family Foundation, Helmsley Charitable Foundation, Larson Charitable Foundation, Wolfson Family Charitable Trust, Charles Rothschild, Selmo Nussenbaum, Miel de Botton (R.M.), the NIH (1R35 GM118043-01 [Maximizing Investigators' Research Award]) (R.P.), Merkin Institute for Translational Research (R.P.), the Israeli Council for Higher Education via the Weizmann Data Science Research Center, and by a research grant from Madame Olga Klein - Astrachan (R.S.). R.M. is the Charles and Louise Gartner Professional Chair. Y.M.B.-O. is an Azrieli Fellow.

25. J.-M. Kim et al., Identification of coronavirus isolated from a patient in Korea with COVID-19. Osong Public Health Res. Perspect. 11, 3-7 (2020).

26. S. Klein et al., SARS-CoV-2 structure and replication characterized by in situ cryo electron tomography. Nat. Commun. 11, 5885 (2020)

27. N. S. Ogando et al., SARS-coronavirus-2 replication in vero E6 cells: Replication kinetics, rapid adaptation and cytopathology. J. Gen. Virol. 101, 925-940 (2020).

28. K. C. Stone, R. R. Mercer, P. Gehr, B. Stockstill, J. D. Crapo, Allometric relationships of cell numbers and size in the mammalian lung. Am. J. Respir. Cell Mol. Biol. 6, 235-243 (1992)

29. J. D. Crapo, B. E. Barry, P. Gehr, M. Bachofen, E. R. Weibel, Cell number and cell characteristics of the normal human lung. Am. Rev. Respir. Dis. 126, 332-337 (1982).

30. $R . K e, C$. Zitzmann, R. M. Ribeiro, A. S. Perelson, Kinetics of SARS-CoV-2 infection in the human upper and lower respiratory tracts and their relationship with infectiousness. bioRxiv [Preprint] (2020). https://doi.org/10.1101/2020.09.25.20201772 (Accessed 30 March 2021).

31. A. Gonçalves et al., Timing of antiviral treatment initiation is critical to reduce SARS-CoV-2 viral load. CPT Pharmacometrics Syst. Pharmacol. 9, 509-514 (2020).

32. R. Milo, R. Phillips, Cell Biology by the Numbers (Garland Science, ed. 1, 2015).

33. H. Y. Chen, M. Di Mascio, A. S. Perelson, D. D. Ho, L. Zhang, Determination of virus burst size in vivo using a single-cycle SIV in rhesus macaques. Proc. Natl. Acad. Sci. U.S.A. 104, 19079-19084 (2007).

34. M. M. Lamers et al., SARS-CoV-2 productively infects human gut enterocytes. Science 369, 50-54 (2020)

35. R. Sender, R. Milo, The distribution of cellular turnover in the human body. Nat. Med. 27, 45-48 (2021)

36. W. Sungnak et al.; HCA Lung Biological Network, SARS-CoV-2 entry factors are highly expressed in nasal epithelial cells together with innate immune genes. Nat. Med. 26, 681-687 (2020)

37. S. Lukassen et al., SARS-CoV-2 receptor ACE2 and TMPRSS2 are primarily expressed in bronchial transient secretory cells. EMBO J. 39, e105114 (2020).

38. C. G. K. Ziegler et al.; HCA Lung Biological Network, SARS-CoV-2 receptor ACE2 is an interferon-stimulated gene in human airway epithelial cells and is detected in specific cell subsets across tissues. Cell 181, 1016-1035.e19 (2020).

39. A. A. Valyaeva, A. A. Zharikova, A. S. Kasianov, Y. S. Vassetzky, E. V. Sheval, Lung epithelial stem cells express SARS-CoV-2 entry factors: Implications for COVID-19. bioRxiv [Preprint] (2020). https://doi.org/10.1101/2020.05.23.107334 (Accessed 9 October 2020).

40. A. S. Iyer et al., Dynamics and significance of the antibody response to SARS-CoV-2 infection. medRxiv [Preprint] (2020). https://doi.org/10.1101/2020.07.18.20155374 (Accessed 9 October 2020).

41. T. F. Rogers et al., Isolation of potent SARS-CoV-2 neutralizing antibodies and protection from disease in a small animal model. Science 369, 956-963 (2020).

42. C. A. Janeway, P. Travers, M. Walport, D. J. Capra, Immunobiology (Garland Science 2001)

43. H. Yao et al., Molecular Architecture of the SARS-CoV-2 virus. Cell 183, 730-738.e13 (2020)

44. B. Turoñová et al., In situ structural analysis of SARS-CoV-2 spike reveals flexibility mediated by three hinges. Science 370, 203-208 (2020)

45. H. P. Taylor, S. J. Armstrong, N. J. Dimmock, Quantitative relationships between an influenza virus and neutralizing antibody. Virology 159, 288-298 (1987).

46. T. C. Pierson, M. S. Diamond, A game of numbers: The stoichiometry of antibody-mediated neutralization of flavivirus infection. Prog. Mol. Biol. Transl. Sci. 129, 141-166 (2015). 
47. M. P. Schön et al., COVID-19 and immunological regulations - From basic and translational aspects to clinical implications. J. Dtsch. Dermatol. Ges. 18, 795-807 (2020)

48. X. Chi et al., Humanized single domain antibodies neutralize SARS-CoV-2 by targeting the spike receptor binding domain. Nat. Commun. 11, 4528 (2020).

49. E. Seydoux et al., Analysis of a SARS-CoV-2-infected individual reveals development of potent neutralizing antibodies with limited somatic mutation. Immunity 53, 98-105.e5 (2020).

50. D. Schub et al., High levels of SARS-CoV-2-specific T cells with restricted functionality in severe courses of COVID-19. JCI Insight 5, e142167 (2020).

51. D. Jacot, G. Greub, K. Jaton, O. Opota, Viral load of SARS-CoV-2 across patients and compared to other respiratory viruses. Microbes Infect. 22, 617-621 (2020).

52. X. He et al., Temporal dynamics in viral shedding and transmissibility of COVID-19. Nat. Med. 26, 672-675 (2020).

53. Y. J. Hou et al., SARS-CoV-2 reverse genetics reveals a variable infection gradient in the respiratory tract. Cell 182, 429-446.e14 (2020).

54. M. Roser, H. Ritchie, E. Ortiz-Ospina, J. Hasell, Coronavirus pandemic (COVID-19). Ou World in Data. https://ourworldindata.org/coronavirus. Accessed 22 March 2021.

55. A. Popa et al., Genomic epidemiology of superspreading events in Austria reveals mutational dynamics and transmission properties of SARS-CoV-2. Sci. Transl. Med. 12, eabe2555 (2020).
56. M. A. Martin, K. Koelle, Reanalysis of deep-sequencing data from Austria points towards a small SARS-COV-2 transmission bottleneck on the order of one to three virions. bioRxiv [Preprint] (2021). https://doi.org/10.1101/2021.02.22.432096 (Accessed 25 February 2021).

57. K. A. Lythgoe et al., Within-host genomics of SARS-CoV-2. bioRxiv [Preprint] (2020). https://doi.org/10.1101/2020.05.28.118992 (Accessed 25 February 2021).

58. S. Duchene et al., Temporal signal and the phylodynamic threshold of SARS-CoV-2. Virus Evol. 6, veaa061 (2020).

59. T. Koyama, D. Platt, L. Parida, Variant analysis of SARS-CoV-2 genomes. Bull. World Health Organ. 98, 495-504 (2020).

60. S. A. Kemp et al.; CITIID-NIHR BioResource COVID-19 Collaboration; COVID-19 Genomics UK (COG-UK) Consortium, SARS-CoV-2 evolution during treatment of chronic infection. Nature 592, 277-282 (2021).

61. B. Choi et al., Persistence and evolution of SARS-CoV-2 in an immunocompromised host. N. Engl. J. Med. 383, 2291-2293 (2020).

62. E. Khatamzas et al., Emergence of multiple SARS-CoV-2 mutations in an immunocompromised host. medRxiv [Preprint] (2021). https://doi.org/10.1101/2021.01.10. 20248871 (Accessed 25 February 2021).

63. S. Jane Flint, V. R. Racaniello, G. F. Rall, A. M. Skalka, L. W. Enquist, Molecular Biology (Principles of Virology, ASM Press, ed. 4, 2015), vol. 1. 